\title{
Disseminated Cutaneous Histoplasmosis in a 30-Year-Old Male HIV- Positive Patient: A Rare Case Report
}

\author{
Vikas Kumar $^{1}$, Astha $^{2 *}$, Yatendra Chahar ${ }^{1}$ and Arti Agrawal ${ }^{1}$ \\ ${ }^{1}$ Sarojini Naidu Medical College, Agra, India \\ ${ }^{2}$ F. H. Medical College, Agra, India \\ *Corresponding author
}

\section{A B S T R A C T}

\begin{tabular}{|l|}
\hline Ke y w or d s \\
Histoplasma \\
capsulatum, \\
Disseminated \\
histoplasmosis, \\
HIV, AIDSs \\
\hline Article Info \\
\hline Accepted: \\
07 May 2019 \\
Available Online: \\
10 June 2019 \\
\hline \hline
\end{tabular}

Histoplasmosis is a systemic mycotic infection caused by Histoplasma capsulatum, a dimorphic fungus. Although histoplasmosis can occur in immunocompetent persons, but immunocompromised individuals, such as people with human immunodeficiency virus (HIV)/acquired immune deficiency syndrome (AIDS), are at high risk of developing disseminated disease. In AIDS patients, mortality may approach 50\%, due to disseminated histoplasmosis, especially in those with severe manifestations. We present a case of disseminated histoplasmosis in a 30-year-old, HIV-positive male patient on antiretroviral treatment, who presented to the Skin outpatient clinic with multiple papulonodular lesions on the face, neck, trunk and ulcers in oral cavity. General complaints included fever, malaise and weight loss, along with hepatosplenomegaly on examination. Investigations revealed pancytopenia, altered liver function and renal dysfunction. CD4 cell count was 72 cells/ $\mu 1$. The patient was started on Amphotericin B for 2 weeks as induction therapy followed by oral itraconazole as maintenance therapy. Histoplasmosis is rarely reported from India, which may be on account of its varied clinical presentation and lack of awareness among dermatologists, hence disseminated histoplasmosis should be considered as a differential diagnosis in an immunocompromised patient presenting with cutaneous lesions, as it is necessary for the early diagnosis and proper management of the patient.

\section{Introduction}

Histoplasmosis is a systemic mycotic infection caused by Histoplasma capsulatum, a dimorphic fungus (1). An American physician, Samuel Darling, who was working in the Canal Zone in Panama, first described histoplasmosis a little over a century ago. He described disseminated form of the disease in a fatal case from Martinique (2). Histoplasmosis occurs in specific endemic areas, such as, North, Central and South America, Africa, India and Southeast Asia while sporadic cases have also been reported in Europe. H. capsulatum is found in the soil of the endemic areas, especially those contaminated with bird and bat droppings (3). The clinical presentation of histoplasmosis ranges from asymptomatic infection or mild respiratory illness to severe disseminated disease. Although histoplasmosis can occur in immunocompetent persons, but 
immunocompromised individuals, such as people with human immunodeficiency virus (HIV)/acquired immune deficiency syndrome (AIDS), are at high risk of developing disseminated disease. In AIDS patients, mortality may approach 50\%, due to disseminated histoplasmosis, especially in those with severe manifestations (4). Although only occasionally reported in the pre-HIV-infection era, it became a public health issue after the AIDS pandemic, and was listed among the AIDS-defining illnesses by Center for Diseases Control and Prevention (CDC) in 1987 (5). Infection occurs when infectious microconidia of Histoplasma capsulatum get aerosolized and are inhaled into the lower airways. In the alveolar space conidia get recognized and phagocytised by the resident macrophages. Inside the macrophages, conidia convert to yeast form - a necessary step in the pathogenesis of histoplasmosis. If cellular immunity is defective, the fungus proliferates and disseminates throughout the body, causing tissue destruction and multi-organ failure, thus leading to progressive dissemination which can be fatal if left untreated (6).

We present a case of disseminated cutaneous histoplasmosis in a 30-year-old, HIV-positive male patient on antiretroviral treatment, who presented to the Skin outpatient clinic with multiple papulonodular lesions on the face, neck, trunk and ulcers in oral cavity.

\section{Case report}

In March, 2019, a 30-year-old male, known case of AIDS (Acquired immunodeficiency syndrome) on antiretroviral treatment, presented to the Skin outpatient clinic of Sarojini Naidu Medical College, Agra with multiple skin colored papulonodular lesions on the face, neck, trunk and ulcers in oral cavity. General complaints included fever, malaise and weight loss, along with hepatosplenomegaly on examination. Investigations revealed pancytopenia, altered liver function and renal dysfunction. CD4 cell count was 72 cells/ $\mu 1$. The patient was then referred to the Microbiology Department for fungal culture of the cutaneous lesions. Culture was performed from skin scrapings collected from the cutaneous lesions, in Sabouraud Dextrose Agar (SDA) tube and incubated at $25^{\circ} \mathrm{C}$. On macroscopic examination, white, cottony colonies were observed after 3 weeks and on microscopic examination of the Lactophenol cotton blue (LPCB) mount, presence of septate hyphae, with tuberculate macroconidia and smoothwalled microconidia were noted, suggestive of histoplasmosis. The conversion from the mold phase to the yeast phase is necessary for accurate diagnosis of $H$. capsulatum. The conversion (dimorphism) test was done by inoculating a portion of the mold colony of suspected Histoplasma on Brain Heart Infusion (BHI) blood agar, and incubating at $37^{\circ} \mathrm{C}$ for the appearance of yeast form of the isolate. On macroscopic examination, the growth of smooth, white yeast-like colonies, was seen on BHI blood agar, incubated at $37^{\circ} \mathrm{C}$. On microscopic examination, budding yeast cells were seen in the Gram stained smear and LPCB mount, thus confirming the diagnosis of histoplasmosis. Fungi belonging to the genus Sepedonium also form similar tuberculate macroconidia but they are not dimorphic and do not form microconidia. Pancytopenia with CD4 count of 72 cells/ $\mu 1$ and altered liver and renal functions, in the setting of AIDS was concerning for disseminated histoplasmosis, and thus blood culture was also done. Blood culture showed H.capsulatum growth, consistent with the suspected diagnosis of disseminated Histoplasma capsulatum infection.

The patient was started on Amphotericin B for 2 weeks as induction therapy, then 
switched over to oral itraconazole as maintenance therapy. The anti-retroviral therapy was also continued with the advice for regular follow-up.

\section{Results and Discussion}

Histoplasmosis is rarely reported from India, which may be on account of its varied clinical presentation and lack of awareness among dermatologists. Histoplasmosis from India, was first reported by Panja and Sen, in 1959 (7). H. capsulatum is believed to be endemic in some East Indian states like West Bengal, where a prevalence of skin positivity of $9.4 \%$ to histoplasmin antigen, was shown by a study (8). A few sporadic cases have been reported from South India as well (9). Histoplasmosis mortality is greater than $33 \%$ in immunosuppressed patients, while in immunocompetent individuals it is approximately 17\% (10) (Fig. 1-3).

Fig.1 Multiple papulonodular lesions on face and neck
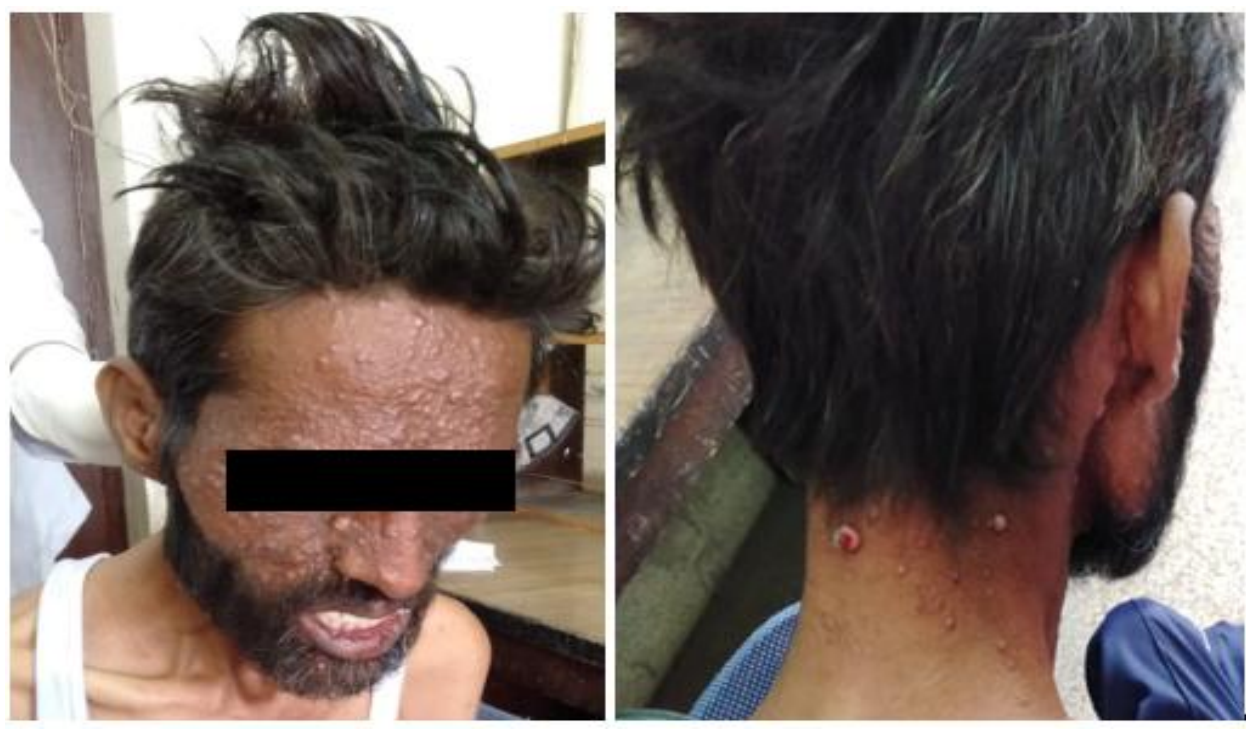

Fig.2 White, cottony colonies on SDA

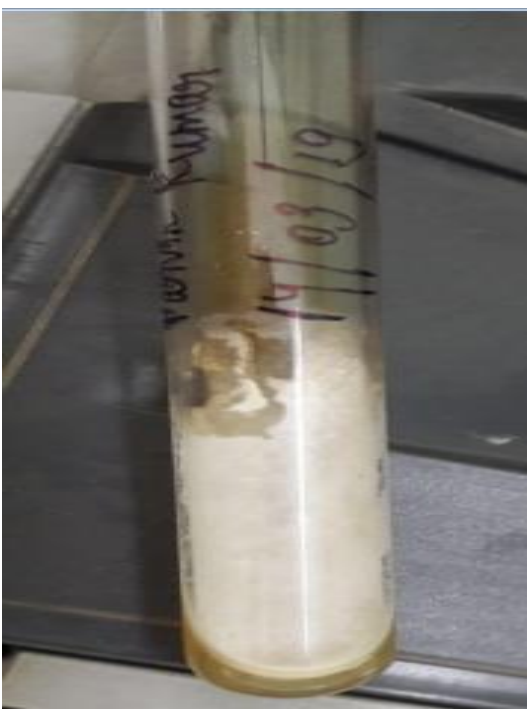


Fig.3 LPCB mount showing hyphae with tuberculate macroconidia

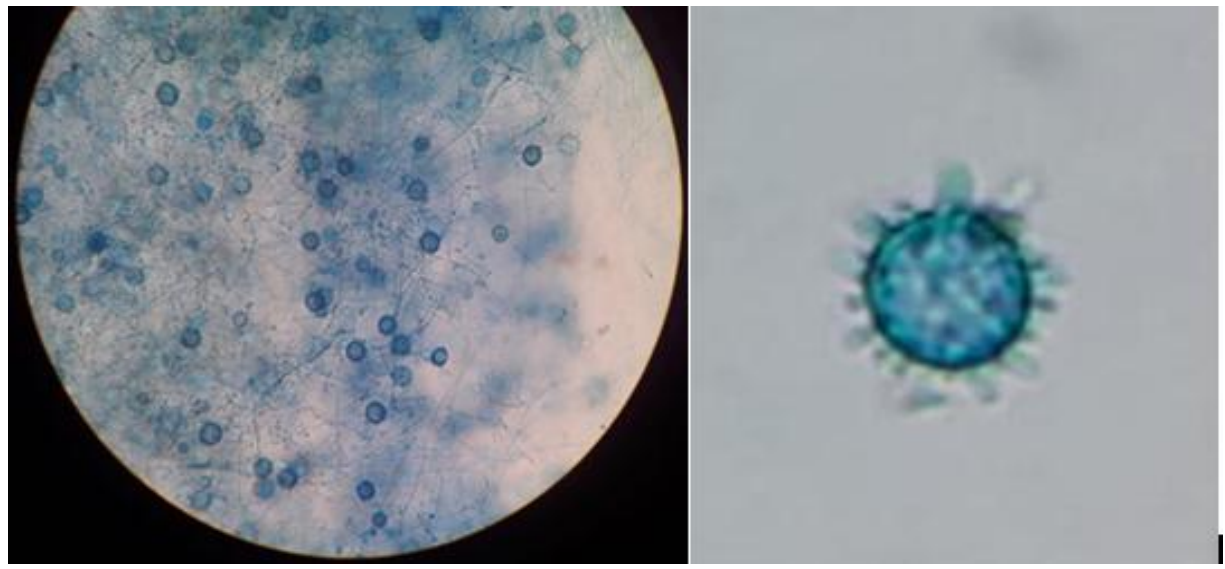

The disseminated disease is the most commonly reported form in immunocompromised individuals and skin lesions are usually the manifestations of disseminated histoplasmosis. Primary cutaneous histoplasmosis is very rare, with cutaneous lesions as the initial presentation of histoplasmosisin about $10 \%$ cases, serving as the marker for AIDS in areas endemic for histoplasmosis (11). In the present case, though there were no signs of pulmonary involvement, the presence of pancytopenia, altered liver function and renal dysfunction, CD4 cell count of 72 cells/ $\mu 1$, hepatosplenomegaly, with multiple papulonodular lesions on the face, neck and ulcers in oral cavity indicate secondary cutaneous histoplasmosis.

Hence, disseminated histoplasmosis should be considered as a differential diagnosis in an immunocompromised patient presenting with cutaneous lesions. After the diagnosis is confirmed, effective and proper antifungal treatment should be timely delivered to the patient, aiming to enhance the clinical recovery.

\section{References}

1. Vidyanath, S., Shameena P M, Sudha $S$, Nair RG. Disseminated histoplasmosis with oral and cutaneous manifestations. J Oral Maxillofac Pathol 2013; 17: 139-42

2. Kauffman, CA., Histoplasmosis: a Clinical and Laboratory Update. Clin Microbiol Rev 2007; 20: 115-132

3. Ashbee, HR., Evans EG, Viviani MA, Dupont B, Chryssanthou E, Surmont I, Tomsikova A, Vachkov $\mathrm{P}$, Enero $\mathrm{B}$, Zala J, Tintelnot $\mathrm{K}$; ECMM Working Group on Histoplasmosis. Histoplasmosis in Europe: report on an epidemiological survey from the European Confederation of Medical Mycology Working Group. Med Mycol. 2008 Feb; 46(1): 57-65.

4. Samayoa, B., Roy M, Cleveland AA, Medina N, Lau-Bonilla D, Scheel CM et al., High Mortality and Coinfection in a Prospective Cohort of Human Immunodeficiency Virus/Acquired Immune Deficiency Syndrome Patients with Histoplasmosis in Guatemala. Am. J. Trop. Med. Hyg. 2017; 97(1): 42-48

5. Zanotti P., Chirico C., Gulletta M., Ardighieri L., Casari S., Quiros Roldan E., Izzo I., Pinsi G., Lorenzin G., Facchetti F., Castelli F. and Focà E. Disseminated Histoplasmosis as AIDS-presentation. Case Report and 
Comprehensive Review of Current Literature. Mediterr J Hematol Infect Dis 2018, 10(1): e2018040 DOI 10.4084/MJHID.2018.040.

6. Knox, KS., and Hage CA. Histoplasmosis. Proc Am Thorac Soc 2010; Vol 7: pp 169-172.

7. Bhagwat $\mathrm{P}$ V., Hanumanthayya K, Tophakhane, R S, Rathod R M. Two unusual cases of histoplasmosis in human immunodeficiency virusinfected individuals. Indian J Dermatol Venereol Leprol 2009; 75: 173-6.

8. Sanyal, M., Thammayya A. Skin sensitivity to histoplasmin in Calcutta and its neighbourhood. Indian $\mathbf{J}$ Dermatol Venereol Leprol 1980; 46:
94-8.

9. Nair, S P., Vijayadharan M, Vincent M. Primaty Cutaneous Histoplasmosis. Indian $\mathrm{J}$ Dermatol Venereol Leprol 2000; 66: 151-3.

10. voss Gonzalez, T., Mattos e Dinato SL, Sementilli A, Romiti N, Beltrame PM, Veiga APR. Atypical presentation of histoplasmosis in an immunocompromised patient. An Bras Dermatol. 2015; 90 (3 Suppl 1): S325.

11. Chande, C., Menon S, Gohil A, Lilani S, Bade J, Mohammad S, Joshi A. Cutaneous histoplasmosis in AIDS. Indian J Med Microbiol 2010; 28:4046

\section{How to cite this article:}

Vikas Kumar, Astha, Yatendra Chahar and Arti Agrawal. 2019. Disseminated Cutaneous Histoplasmosis in a 30-Year-Old Male HIV-Positive patient: A Rare Case Report. Int.J.Curr.Microbiol.App.Sci. 8(06): 722-726. doi: https://doi.org/10.20546/ijcmas.2019.806.085 